\title{
Efecto del aumento de la oferta educativa en la escolaridad y el trabajo adolescente en el Perú rural ${ }^{1}$
}

\section{Brian Daza}

Centro de Investigación de la Universidad del Pacífico

bdaza@alum.mit.edu

Recibido: 06/03/2019

Aprobado: 01/04/2020

1. Esta investigación se desarrolló en el marco del Primer Concurso de Becas para Jóvenes Investigadores (2018), organizado por la Sociedad de Investigación Educativa Peruana (SIEP) con el apoyo de Unicef. 


\title{
Efecto del aumento de la oferta educativa en la escolaridad y el trabajo adolescente en el Perú rural
}

\begin{abstract}
Resumen
Durante los años 2004 y 2017, se crearon 1809 servicios educativos de nivel secundario en el área rural, es decir, aproximadamente, $40 \%$ de la oferta educativa total del nivel secundario en 2017, que correspondió a 4230 servicios. Esta investigación busca identificar los efectos de la expansión de la cobertura educativa de nivel secundaria en la escolaridad y la participación laboral adolescente en el área rural. La metodología empleada se basa en un método de diferencias en diferencias generalizado, y utiliza principalmente información de la Encuesta Nacional de Hogares 2004-2017 y el Padrón de Instituciones Educativas del Ministerio de Educación del año 2017. A partir de ello, se identifica que el aumento de la cobertura educativa en el área rural ha tenido impacto positivo en la matrícula escolar, en los años de educación alcanzados y en la reducción de la prevalencia del empleo adolescente. Los resultados muestran mayor impacto en la población femenina y en los departamentos con mayor población rural.
\end{abstract}

Palabras clave: Escolaridad, trabajo, adolescencia, educación secundaria, ruralidad

\section{Effect of the increase of the educational supply on schooling and adolescent work in rural Peru}

\begin{abstract}
In the period 2004-2017, 1809 secondary-level educational services were created in the Peruvian rural area, 40\% of the total of active secondary services in 2017 (4230). This article attempts to identify this expansion's effects on schooling and teenage labor participation in rural areas. The study bases on a generalized difference in differences method, relying on information from the National Household Survey 2004-2017 and the Register of Educational Institutions of the Ministry of Education in 2007. The analysis shows that the increase in educational services in the rural area has had a positive impact on school enrollment, on the years of education attained, and on the reduction of teenage labor. Results showed that the impacts are greater in the case of female population and in the departments with the largest rural population.
\end{abstract}

Keywords: Schooling, work, adolescence, secondary education, rurality 


\section{Introducción}

De acuerdo con el Sistema de Información de Tendencias Educativas en América Latina (2010), a partir de encuestas de hogares de la región, se registra una fuerte interrelación entre el empleo en adolescentes ( 13 a 17 años) y la no concurrencia a centros educativos en los países observados, con excepción de Brasil y Bolivia. En el caso del año 2008, los países con menor tasa de empleo adolescente - Chile $(3,4 \%)$ y Argentina $(6,6 \%)$ - son también aquellos con menores tasas de exclusión del sistema educativo en este grupo etario $(6,4 \%$ y $9,5 \%$, respectivamente). Por su parte, en Guatemala, el país con mayor población adolescente en el mercado laboral (42,5\%), el 39\% de los adolescentes no estudia. En este estudio, en el caso del Perú, se reporta que el porcentaje de adolescentes fuera del sistema educativo es de aproximadamente $26 \%$ y que la tasa de empleo adolescente es de $31 \%$. La elevada intersección entre ambos grupos de riesgo se evidencia en el hecho de que, de acuerdo con el estudio, la tasa de empleo entre los adolescentes que están fuera del sistema educativo es entre dos y diez veces mayor que entre los adolescentes que sí estudian.

Según los resultados de la Encuesta Nacional Especializada en Trabajo Infantil (ETI 2015),

en el área rural [peruana], el 52,3\% de la población de 5 a 17 años participaron en la actividad económica; mientras que en el área urbana fue $16,2 \%$. Por grupos de edad, el $12,1 \%$ de la población de 5 a 9 años trabaja, seguido del $29,3 \%$ de 10 a 13 años y el 40,5\% de 14 a 17 años laboran. (INEI, 2016a)

La educación debería permitir a los niños y los adolescentes alcanzar mayores niveles de bienestar social, satisfacción individual, y perspectivas de empleo y desarrollo personal en el futuro. Si los servicios educativos no cumplen ese rol, por ausencia o incapacidad, resultarán necesarias acciones de política. Adicionalmente, la sociedad podría verse impactada negativamente en la medida en que el trabajo infantil y adolescente prevalezcan. En un estudio enfocado en Zambia, por ejemplo, Nielsen (1998) menciona que este fenómeno produce una pérdida de entre $1 \%$ y $2 \%$ del PBI, lo cual resulta preocupante si se considera que afecta principalmente al área rural, que es donde se ubican los hogares más vulnerables.

En el Perú, tanto la deserción escolar como el empleo adolescente son fenómenos vigentes. En el área rural, este problema no solamente tiene una mayor magnitud, sino que adquiere mayores dimensiones de complejidad al interactuar con las características propias del entorno, entre las que se incluyen también las diferencias en la calidad y la oferta del servicio educativo. Cabe anotar que existen iniciativas que reconocen la urgencia de resolver las deficiencias del sistema educativo. De hecho, en el año 2006, se presentó al país el Proyecto Educativo Nacional al 2021 (Consejo Nacional de Educación, 2006). 
El aumento y la mejora de la oferta educativa en el sector rural, que es donde la atención del sistema educativo ha sido menor, son claves para cumplir dos de los objetivos estratégicos del proyecto: "OE1: Oportunidades y resultados educativos de igual calidad para todos" y "OE6: Una sociedad que educa a sus ciudadanos y los compromete con su comunidad". En el mismo documento, la Política $\mathrm{N}^{\circ} 2.2$ define como una de las principales medidas a adoptar

la aplicación continua y sistemática de modalidades flexibles que respondan con calidad y pertinencia a las condiciones reales de vida, cultura y trabajo en zonas rurales, enfaticen el desarrollo de competencias productivas, emprendedoras y de ciudadanía, y que puedan ser replicadas. (Consejo Nacional de Educación, 2006)

Por lo menos desde el 2001, el sistema educativo peruano debería garantizar por ley ${ }^{2}$ el acceso y atención adecuada a los niños, niñas y adolescentes en el área rural. No obstante, la cobertura del servicio educativo, sobre todo en el área rural, aún no es universal.

En la Figura 1, se presenta un resumen gráfico del incremento de la cobertura educativa en el área rural peruana. El indicador utilizado es el número de servicios educativos rurales de nivel secundaria operativos en cada distrito por cada cien habitantes de entre diez a diecinueve años. Comparando el año inicial y el último del período analizado, se pueden destacar dos hechos. Por un lado, la cobertura ha mejorado sustancialmente en varios distritos del Perú, mientras que en otros el cambio no es perceptible. Por otro lado, la dispersión del cambio no sigue un patrón geográfico evidente.

2. Dicho deber es planteado en la Ley 27558, Ley de Fomento de la Educación de las Niñas y Adolescentes Rurales, 23 de noviembre de 2001. 
Figura 1. Servicios educativos de nivel secundaria por cada 100 habitantes de entre 10 a 19 años en el área rural
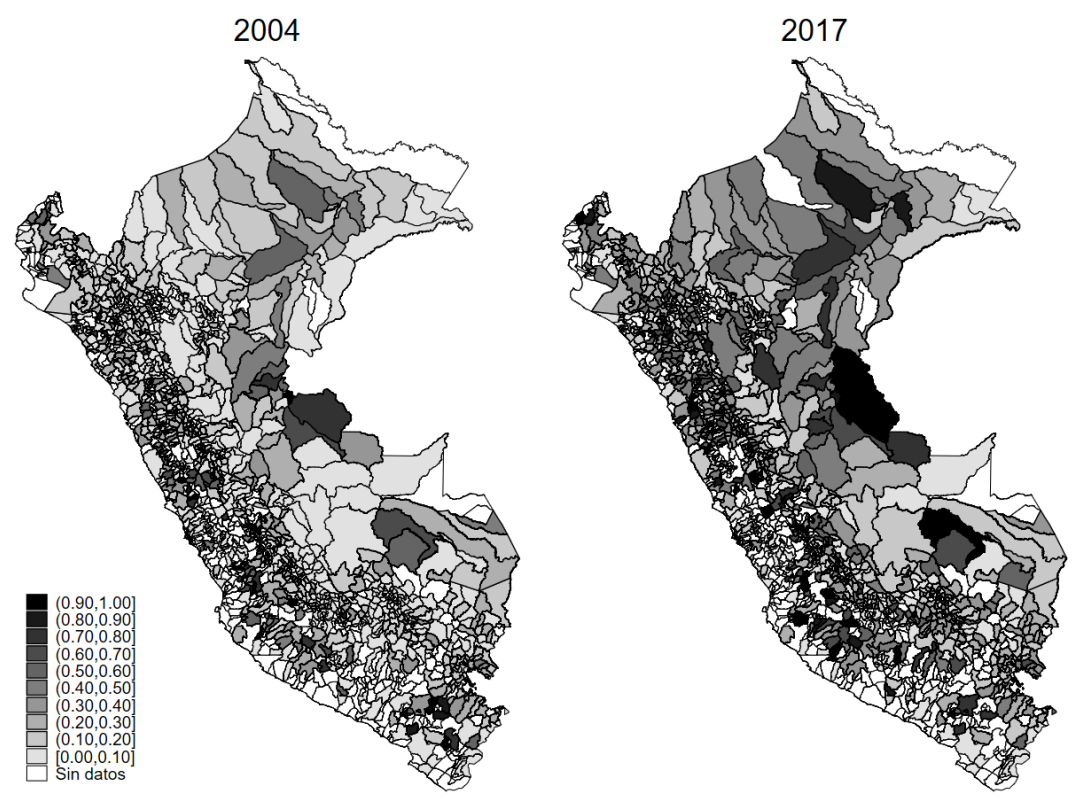

Fuente: Elaboración propia, con datos del Padrón de Instituciones Educativas, ENAHO 2004 y 2017, y CPV 2007.

Adicionalmente, en la Tabla 1, se presenta un resumen del cambio observado entre los años 2004 y 2017 de la oferta educativa en el Perú, e indicadores de escolaridad y empleo para adolescentes del área rural. En conjunto, además del aumento de la oferta del servicio educativo en el área rural, se puede ver que la prevalencia del trabajo adolescente es elevada y que la participación de los adolescentes en la educación no es universal. Si bien los indicadores educativos muestran una mejora, también ha aumentado la proporción de adolescentes en el área rural que trabajan, aunque su jornada laboral es menor en promedio. Resulta relevante, entonces, analizar los efectos causales de políticas como el aumento de la oferta educativa en el sector rural, en la participación de los adolescentes en el mercado laboral y en sus decisiones de escolaridad. 
Tabla 1. Estadísticas descriptivas

\begin{tabular}{|c|c|c|c|c|c|c|c|c|}
\hline & \multicolumn{2}{|c|}{2004} & \multicolumn{2}{|c|}{2017} & \multicolumn{2}{|c|}{ Diferencia } & \multicolumn{2}{|c|}{$\begin{array}{l}\text { Pooled 2004- } \\
2017\end{array}$} \\
\hline & Media & $\begin{array}{l}\text { Error } \\
\text { estándar }\end{array}$ & Media & $\begin{array}{c}\text { Error } \\
\text { estándar }\end{array}$ & Media & $\begin{array}{l}\text { Error } \\
\text { estándar }\end{array}$ & Media & $\begin{array}{l}\text { Error } \\
\text { estándar }\end{array}$ \\
\hline \multicolumn{9}{|c|}{ Oferta de servicios educativos } \\
\hline Total & 7913 & -- & 12445 & -- & 4532 & -- & & \\
\hline Área urbana & 6154 & -- & 8877 & -- & 2723 & -- & & \\
\hline Área rural & 1759 & -- & 3568 & -- & 1809 & -- & & \\
\hline $\begin{array}{l}\text { Por cada cien personas } \\
\text { de } 10-19 \text { años en el } \\
\text { área rural }\end{array}$ & 0,139 & 0,008 & 0,384 & 0,01 & 0,245 & 0,013 & 0,227 & 0,005 \\
\hline \multicolumn{9}{|c|}{ Escolaridad y empleo en adolescentes rurales ( 14 a 17 años) } \\
\hline \multicolumn{9}{|c|}{ Población Femenina } \\
\hline $\begin{array}{l}\text { Años de educación } \\
\text { aprobados }\end{array}$ & 9,947 & 0,092 & 11,478 & 0,053 & 1,530 & 0,106 & 10,775 & 0,029 \\
\hline $\begin{array}{l}\text { Matrícula en un centro } \\
\text { de enseñanza }\end{array}$ & $64,60 \%$ & 0,019 & $82,00 \%$ & 0,012 & $17,40 \%$ & 0,022 & $72,76 \%$ & 0,006 \\
\hline $\begin{array}{l}\text { Asistencia regular a un } \\
\text { centro de enseñanza }\end{array}$ & $93,50 \%$ & 0,013 & $91,20 \%$ & 0,011 & $-2,30 \%$ & 0,017 & $92,18 \%$ & 0,004 \\
\hline $\begin{array}{l}\text { Participación en el } \\
\text { mercado de trabajo }\end{array}$ & $44,60 \%$ & 0,019 & $53,10 \%$ & 0,016 & $8,50 \%$ & 0,025 & $52,47 \%$ & 0,005 \\
\hline $\begin{array}{l}\text { Horas de trabajo a la } \\
\text { semana }\end{array}$ & 26,199 & 0,685 & 18,961 & 0,509 & $-7,238$ & 0,853 & 22,764 & 0,203 \\
\hline \multicolumn{9}{|l|}{ Población masculina } \\
\hline $\begin{array}{l}\text { Años de educación } \\
\text { aprobados }\end{array}$ & 10,404 & 0,076 & 11,411 & 0,047 & 1,007 & 0,089 & 10,878 & 0,025 \\
\hline $\begin{array}{l}\text { Matrícula en un centro } \\
\text { de enseñanza }\end{array}$ & $72,40 \%$ & 0,017 & $84,30 \%$ & 0,011 & $11,90 \%$ & 0,02 & $77,39 \%$ & 0,005 \\
\hline $\begin{array}{l}\text { Asistencia regular a un } \\
\text { centro de enseñanza }\end{array}$ & $91,70 \%$ & 0,012 & $92,20 \%$ & 0,009 & $0,60 \%$ & 0,015 & $92,33 \%$ & 0,004 \\
\hline $\begin{array}{l}\text { Participación en el } \\
\text { mercado de trabajo }\end{array}$ & $57,90 \%$ & 0,017 & $62,30 \%$ & 0,013 & $4,40 \%$ & 0,022 & $63,38 \%$ & 0,005 \\
\hline $\begin{array}{l}\text { Horas de trabajo a la } \\
\text { semana }\end{array}$ & 26,653 & 0,571 & 20,657 & 0,475 & $-5,996$ & 0,743 & 23,471 & 0,193 \\
\hline
\end{tabular}

*Nota: Existen 2057 servicios educativos. Entre ellos, 662 se ubican en el área rural, para los cuales no se pudo identificar la fecha de incorporación al padrón y que no se cuentan en este cuadro. Los años de educación se cuentan considerando tres años de educación preescolar. La asistencia regular incluye solamente a los adolescentes que registran matrícula en un centro educativo.

Fuente: Elaboración propia, con datos del Padrón de Instituciones Educativas 2017, ENAHO 2004-2007 y CPV 2007 
En este trabajo, se encuentra evidencia de que la oferta educativa ha tenido influencia en la configuración del empleo adolescente y en la escolaridad, medida en años de educación lograda. Adicionalmente, las heterogeneidades entre el nivel de ruralidad de las diferentes regiones del Perú y el género influyen en la respuesta de las variables de interés. Los resultados muestran que la expansión de la oferta educativa ha sido importante para mejorar los indicadores de logros educativos y trabajo adolescente, sobre todo, en la población femenina. No obstante, parece no haber tenido la misma eficacia en regiones con mayor población rural y en la población masculina.

La presente investigación se divide en cinco secciones. La primera presenta una revisión de la literatura sobre el trabajo adolescente e infantil y la participación escolar, y su vínculo con la oferta educativa. La segunda sección describe la metodología empírica. La tercera presenta los resultados, mientras que la cuarta plantea la discusión y las implicancias de dichos resultados a nivel académico. En la última sección, se desarrollan las conclusiones del estudio.

\section{Revisión de literatura}

Tal y como señalan Beegle, Dehejia, y Gatti (2009), tanto en la arena política como en la académica, se reconoce que el trabajo adolescente e infantil constituye un problema con múltiples consecuencias potenciales. Existen opiniones serias y evidencia que respaldan que genera efectos negativos en el nivel educativo, el desempeño laboral futuro y la salud de los menores. Se estima que los beneficios de eliminar el trabajo infantil serían sustantivos (International Programme on the Elimination of Child Labour, 2004). Sin embargo, la evidencia sobre el mecanismo causal es limitada. La mayor parte de los estudios empíricos existentes que asocian el trabajo infantil y la participación escolar muestran una fuerte relación negativa, pero no concluyen una sola dirección en la relación causa-efecto. Algunos estudios refuerzan dos posibles direcciones de causalidad a nivel teórico y empírico.

A partir de una perspectiva teórica y usando como referencia la función de ingresos de Mincer, se puede considerar que las decisiones de escolaridad y de trabajo forman parte del mismo proceso de optimización, en el cual interfieren los retornos esperados de la educación y del tiempo de experiencia potencial. Desde este punto de vista, existiría un trade-off, puesto que los años de educación y los años de experiencia laboral (que podrían iniciarse en el trabajo adolescente e infantil) se determinarían en conjunto, en función de cómo los hogares perciben que esta decisión influye en sus perspectivas de ingresos. La evidencia empírica para esta hipótesis, como discutiremos brevemente, no es clara o unidireccional, y depende en gran medida del contexto.

Aún desde una perspectiva teórica, existe la posibilidad de que esta relación sea aún más compleja, especialmente, para las personas de bajos recursos. El estudio de Ravallion y Wodon (2000), una de las referencias más citadas, ejemplifica esto. Mediante un subsidio focalizado relacionado con la inscripción escolar en el área rural en Bangladesh, los autores identificaron que este tipo de incentivos genera un aumento de la escolaridad, pero un efecto 
ambiguo en el trabajo adolescente e infantil. Esta ambigüedad se alimenta de diversos factores que están empezando a ser analizados por la literatura. Por ejemplo, los padres pueden subestimar los beneficios de la educación o los resultados alcanzados por sus hijos. Dicho comportamiento puede conducir a una subinversión en educación, que puede ser muy sensible a cambios en las expectativas de retornos futuros (Dizon-Ross, 2019). Desde otro ángulo, es posible que la variable educativa se relacione con las denominadas "trampas de pobreza"', en tanto es necesario tener cierto nivel acumulado de años de escolaridad para empezar a percibir mejoras en productividad o retornos por cada año adicional de educación. Es decir, al inicio, la educación puede no tener retornos sobre el nivel de ingresos que las personas pueden generar. Este escenario podría agravarse por el hecho de que la educación a la que tienen acceso las personas en situación de pobreza suele ser de muy baja calidad, y, por lo tanto, no ofrecer perspectivas de mejora para la infancia y adolescencia.

En el Perú, uno de los estudios más recientes sobre las decisiones de inserción laboral de los jóvenes rurales (Boyd, 2014) muestra que la decisión de estudiar de los jóvenes y adolescentes se encuentra muy ligada a la decisión de empezar a trabajar. Además, considerando el elevado porcentaje de realización de trabajo familiar no remunerado (aproximadamente, 40\%), existe fuerte evidencia de que esta decisión se realiza dentro del hogar y no a nivel de individuo. El estudio de Boyd da cuenta de que la dinámica rural es diferente a la urbana. En el primer escenario, el desempleo es menor; y la actividad laboral, mayor.

La literatura ha explorado el hecho de que factores exógenos de oferta influyen en la decisión de escolaridad. Estas restricciones de oferta pueden elevar significativamente los costos de escolaridad, y, por lo tanto, conducir a que muchos niños y adolescentes no se beneficien de las potenciales ganancias que les podría otorgar la educación. Para el caso de Zambia, Nielsen (1998) ha encontrado evidencia de que restricciones de acceso a centros educativos - como la distancia, la infraestructura de transporte y los costos asociados- están estrechamente relacionadas con las decisiones de escolaridad y empleo. Para los casos en los que opera esta restricción, se encuentra una fuerte correlación negativa entre la asistencia a la escuela y el trabajo. De acuerdo con Nielsen, la poca tradición educativa de la familia y el escaso acceso a centros educativos son las variables con mayor capacidad para explicar la falta de participación en el sistema educativo.

La investigación realizada por Emerson y Souza (2007) en Brasil muestra resultados heterogéneos en la decisión de escolaridad y empleo de los hijos adolescentes a partir de su género, y el poder relativo del padre y de la madre al interior de la familia. Por ejemplo, una mayor capacidad económica del padre puede estar más relacionada con la reducción del trabajo adolescente en general, mientras que un aumento de la capacidad adquisitiva de la madre puede tener mucho mayor impacto en las decisiones de escolaridad de las hijas, en comparación con lo que hubiera producido el mismo aumento en el caso del padre.

3. El tema de las trampas de pobreza en general y su vínculo con la educación ha sido discutido por Dasgupta (2007); Knight, Shi y Quheng (2010); Van der Berg et al. (2011), entre otros. 
Del mismo modo, en una investigación realizada en Colombia, Urueña, Tovar y Castillo (2009) muestran que los menores con mayor riesgo de trabajar a costa de no asistir a la escuela son los hijos mayores, los hijos varones, los hogares con jefe de hogar poco educado, los hogares en que el jefe de hogar es varón, los menores en el área rural, los menores en hogares con numerosos integrantes, y los que viven en hogares con mayor tasa de ocupación de los adultos.

Volviendo al caso peruano, Cueto (2004) señala que un problema fundamental de la escuela estatal, con mayor gravedad en el área rural, se relaciona al hecho de que esta no se adapta a las necesidades de los estudiantes. Al contrario, es "Darwiniano, en el sentido de que [...] espera que los estudiantes se adapten al medio escolar" (Cueto, 2004). En ese sentido, los estudiantes que ya tienen las características necesarias para "sobrevivir" son quienes acaban teniendo mayor rendimiento. Cueto identifica un resultado interesante: el mal rendimiento de los estudiantes no condiciona de manera significativa que abandonen la escuela. Ello se manifiesta en las altas cifras de estudiantes del área rural que han repetido varias veces de grado en años seguidos. Incluso, en las evaluaciones realizadas por Cueto (2004), se observa que estos estudiantes mejoran su rendimiento en comparación con los desertores. A su vez, el autor halla que el rendimiento académico y la deserción escolar se explican fundamentalmente por la poca o nula educación formal de los padres, el hablar una lengua indígena, la educación en aulas multigrado, la mayor edad relativa, la falta de materiales pedagógicos, el género femenino, el tener características físicas favorables para el trabajo, y la situación de pobreza de las familias. Estos factores se relacionan profundamente con la necesidad o el deseo de trabajar.

Alcázar, Rendón y Wachtenheim (2002) habían encontrado evidencias de trade-offs en el área rural entre empleo adolescente y escolaridad. En este caso, los determinantes cruciales eran el ingreso y educación de los padres, la composición familiar, y la existencia de círculos viciosos y virtuosos intergeneracionales.

En un estudio posterior, Alcázar (2009) se aproxima de manera más directa a nuestro objetivo de investigación enfocándose en la deserción escolar en las escuelas secundarias rurales del Perú. Su estudio, en primer lugar, confirma que la pobreza, las variables relacionadas a la misma, los factores familiares, y la percepción de la calidad e impacto de la educación son variables fundamentales para explicar la deserción escolar a través de su relación con el empleo adolescente. Sin embargo, el hallazgo más importante es que existen evidencias de que la carencia de centros educativos próximos es un factor crucial para determinar si un joven o adolescente recibe servicios educativos. En otras palabras, las características de los jóvenes rurales en localidades donde hay oferta de educación secundaria son estadísticamente similares a las de aquellos que residen donde no hay oferta. Por un lado, a diferencia de Cueto, Alcázar encuentra que el historial educativo de los jóvenes sí se relaciona con la probabilidad de deserción; es decir, un mayor atraso escolar puede conducir a que los jóvenes opten por dejar de estudiar. Por otro lado, similar a lo que plantea Cueto, Alcázar identifica que este fenómeno se refuerza porque los horarios, programas y demás características de las instituciones educativas no responden a las necesidades específicas de los educandos. 
Siguiendo esa línea, Chacaltana (2012) resalta el papel de la distancia a los centros educativos y el déficit de calidad existente en la deserción escolar en el área rural. Señala, además, que en este ámbito es relevante la dependencia con el ciclo agrícola, la predominancia del trabajo familiar no remunerado, la diversificación de actividades (que implica elevada movilidad geográfica temporal) y el énfasis en cuanto a las diferencias de género.

Las comunidades rurales en el Perú son altamente vulnerables ante shocks externos, particularmente, desastres naturales. La costa norte, por ejemplo, es vulnerable ante eventos como el fenómeno de El Niño. Por su parte, varias comunidades de la sierra y la selva son particularmente sensibles ante variaciones drásticas de precipitación pluvial, es decir, sequías o lluvias intensas que ocasionan huaicos o inundaciones. Marchetta, Sahn y Tiberti (2018), en un estudio para Madagascar, encuentran que el nivel de lluvias y la presencia de ciclones tienen efectos significativos sobre las decisiones de escolaridad y trabajo. No obstante, el grado de vulnerabilidad ante este y otro tipo de shocks puede ser amortiguado por variables como acceso al crédito o posesión de activos durables (Beegle, Dehejia, y Gatti, 2006).

La lejanía o la inaccesibilidad de los centros educativos conduce a que el uso de servicios educativos requiera el transporte de grandes distancias. Tanto para los propios niños y adolescentes como para sus padres, el que los hijos tengan que desplazarse lejos del hogar disuade en gran medida su asistencia regular y la culminación de sus estudios en favor del trabajo en el hogar o en la comunidad (Li y Liu, 2014). Esto se evidencia particularmente en espacios en los que los estudiantes corren riesgo por peligros sociales o ambientales.

Otras investigaciones dan cuenta de que la presencia de servicios educativos facilita el involucramiento de los padres en la educación de sus hijos, lo cual constituye una ventaja adicional para el desempeño educativo (Izzo, Weissberg, Kasprow, y Fendrich, 1999). Li y Liu (2014); Song, Appleton, y Knight (2006); Jacoby y Mansuri (2011) muestran que la mayor accesibilidad al sistema educativo, como consecuencia del aumento de establecimientos educativos, conduce a mejoras en la participación escolar y en los logros educativos (única o mayormente) de las mujeres. Estos hallazgos son consistentes con el argumento de que las diferencias en los costos de oportunidad y en las dificultades asociadas a estudiar en un entorno lejano al familiar tienen impacto y este es mayor en el caso de las mujeres (Song et al., 2006; Jacoby y Mansuri, 2011).

\section{Metodología}

La oferta educativa en el nivel primaria está muy extendida y aparentemente en cantidad razonable, dada la práctica universalidad en la conclusión de dicho nivel. La oferta en el nivel secundario es menor, así como los niveles de conclusión, sobre todo, en el sector rural. Sin embargo, de acuerdo con la información del sistema de Estadísticas de la Calidad Educativa (Escale) del Ministerio de Educación, en el período 2004-2017, se crearon 4532 instituciones educativas de nivel secundario, de las cuales 1809 se ubican en el área rural. Considerando que el total de servicios educativos de nivel secundario activos 
en el área rural fue de 4230 en 2017, observamos que, en términos de atención, la oferta educativa en este nivel para el área rural ha aumentado en más de 70\% en los últimos 10 años. Esto quiere decir que el $40 \%$ de la oferta educativa activa actual del nivel secundario en el área rural es de creación reciente.

En las siguientes secciones, indagaremos en si este aumento en la oferta educativa del nivel secundaria en el área rural ha tenido efectos sobre la escolaridad y la participación laboral de los adolescentes en el área rural.

\section{Datos}

La información sobre la prevalencia de trabajo adolescente y la participación en el sistema educativo - que son las variables dependientes por explicar-se obtiene a partir de la integración de las bases de datos de la Encuesta Nacional de Hogares entre los años 2004 y 2017. La base de datos final constituye un pooled cross-section que integra información de los módulos de vivienda, educación, empleo y salud. El área considerada rural en este estudio corresponde a los estratos 6, 7 y 8 de la encuesta, es decir, la población que habita en centros poblados de menos de 400 viviendas y en las áreas de empadronamiento rural clasificadas por el Instituto Nacional de Estadística. A partir de la información de la Encuesta, se estimó el porcentaje de la población que habitaba en área rural en cada departamento y año.

La variable de tratamiento se construyó a partir del Padrón de Instituciones Educativas que ofrece Escale, que detalla la fecha de creación o registro de cada servicio; su ubicación geográfica específica; y algunas variables de interés, como el nivel de ruralidad de acuerdo con la clasificación del Ministerio de Educación en Escale. Según esta clasificación, se considera servicio educativo rural a aquel que está ubicado en un centro poblado con menos de 500 habitantes, excluidos los que se encuentran en una capital de distrito, provincia o departamento.

La denominación de "rural" para una institución educativa es más exigente que la definición de ruralidad que estamos usando para las comunidades a partir de Enaho. No obstante, usar esa clasificación ofrece la ventaja de que podemos esperar mayor homogeneidad entre las instituciones educativas que estaríamos contabilizando y, además, mantendremos homogeneidad con la clasificación censal del Ministerio de Educación.

La síntesis entre la información de Enaho y del padrón se hizo a nivel de distrito. A partir del padrón, se obtuvo el total de servicios educativos rurales de nivel secundario que había en cada distrito en cada año de la muestra. La cantidad de servicios educativos existentes en el distrito se dividió entre el total de la población rural en el distrito de edades entre diez y diecinueve años, de acuerdo con las proyecciones poblacionales elaboradas por el INEI (2016b) ${ }^{4}$. Sobre esta

4. Las proyecciones de población estaban disponibles para el período de 2005 a 2015. Para el año 2017, se usó la información proveniente del Censo Nacional de Población y Vivienda de 2017 (INEI 2017). La información para los años 2004 y 2016 se completó a partir del cálculo de la tasa de crecimiento promedio de la población a nivel de distrito. Se realizó, además, un ajuste de la proporción de la población en el área 
base, la variable usada para medir la oferta educativa se expresó de la siguiente manera: "Cantidad de instituciones educativas de nivel secundaria en el área rural del distrito por cada cien habitantes de entre diez y diecinueve años"s.

Tabla 2. Características socioeconómicas y oferta educativa*

\begin{tabular}{|c|c|c|c|c|c|c|c|c|c|c|}
\hline & & & edia p & cuan & es del in & idicador & de ofert & ta educa & tiva $(x=\varepsilon$ & \\
\hline & $\begin{array}{c}\text { Media } \\
\text { agregada }\end{array}$ & 1 & 2 & 3 & 4 & 5 & 6 & 7 & 8 & $\rho^{\dagger}$ \\
\hline $\begin{array}{l}\text { Indicador } \\
\text { de oferta } \\
\text { educativa }\end{array}$ & 0,181 & 0,001 & 0,039 & 0,079 & 0,117 & 0,160 & 0,215 & 0,292 & 0,544 & 1,00 \\
\hline $\begin{array}{l}\text { Proporción } \\
\text { masculina }\end{array}$ & $52,46 \%$ & $54,18 \%$ & $53,09 \%$ & $52,50 \%$ & $50,22 \%$ & $52,46 \%$ & $52,88 \%$ & $52,85 \%$ & $51,46 \%$ & $-0,01$ \\
\hline Edad & 15,437 & 15,428 & 15,452 & 15,416 & 15,470 & 15,442 & 15,413 & 15,449 & 15,429 & 0,00 \\
\hline Falta de agua & $54,26 \%$ & $57,95 \%$ & $63,83 \%$ & $53,65 \%$ & $56,73 \%$ & $55,40 \%$ & $48,28 \%$ & $52,59 \%$ & $45,70 \%$ & $-0,09$ \\
\hline $\begin{array}{l}\text { Falta de } \\
\text { electricidad }\end{array}$ & $42,72 \%$ & $46,36 \%$ & $51,36 \%$ & $46,53 \%$ & $40,02 \%$ & $43,82 \%$ & $37,39 \%$ & $40,46 \%$ & & $-0,08$ \\
\hline $\begin{array}{l}\text { Jefe de hogar } \\
\text { con baja } \\
\text { escolaridad }\end{array}$ & $72,07 \%$ & $70,59 \%$ & $75,38 \%$ & $74,23 \%$ & $75,35 \%$ & $71,28 \%$ & $71,18 \%$ & $70,76 \%$ & & $-0,05$ \\
\hline $\begin{array}{l}\text { Jefe de hogar } \\
\text { masculino }\end{array}$ & $87,42 \%$ & $86,95 \%$ & $88,28 \%$ & $86,75 \%$ & $87,33 \%$ & $88,46 \%$ & $87,12 \%$ & $88,57 \%$ & $85,87 \%$ & $-0,01$ \\
\hline $\begin{array}{l}\text { Número de } \\
\text { hijos }\end{array}$ & 3,570 & 3,660 & 3,676 & 3,624 & 3,531 & 3,625 & 3,506 & 3,568 & 3,372 & $-0,05$ \\
\hline $\begin{array}{l}\text { Lengua } \\
\text { materna } \\
\text { indígena }\end{array}$ & $29,86 \%$ & $27,37 \%$ & $17,41 \%$ & $30,07 \%$ & $30,22 \%$ & $34,96 \%$ & $38,17 \%$ & $30,70 \%$ & $29,93 \%$ & 0,02 \\
\hline Observaciones & 47.391 & 6346 & 5216 & 5428 & 5391 & 5840 & 5837 & 6490 & 6845 & 47.391 \\
\hline
\end{tabular}

*Nota: Las medias han sido calculadas teniendo en cuenta los factores de expansión y el diseño muestral de la Encuesta Nacional de Hogares.

$\dagger \rho$ : representa la correlación de cada variable con el indicador de oferta educativa. Estas correlaciones consideran los factores de expansión poblacional, la estratificación y la primera unidad de muestreo de Enaho. Dichas correlaciones han sido calculadas mediante el procedimiento de Winter (2001).

Fuente: Elaboración propia, con datos del Padrón de Instituciones Educativas 2017, Enaho 2004-2007 y CPV 2007

rural de cada distrito a partir de las proporciones censales, según el Censo Nacional de Población y Vivienda de 2007.

5. Es necesario mencionar que no se pudo establecer la fecha de fundación o registro de 662 instituciones educativas de nivel secundaria en el área rural. Debido a que se contaba con la información de su ubicación geográfica, se contabilizó como variable de control el número de este tipo de instituciones que tiene cada distrito ponderado por la proyección de población distrital rural entre diez y diecinueve años. 


\section{Estrategia empírica}

La creación de servicios educativos de nivel secundario se puede considerar exógena en el área rural, en línea con lo encontrado por Alcázar (2009). Dicho estudio indica que las características de los jóvenes que viven en zonas rurales donde no hay oferta de educación secundaria son estadísticamente similares a las de aquellos que viven en áreas rurales en las que sí la hay. En la Tabla 2 de este documento, se verifica que no hay diferencias entre las variables socioeconómicas condicionales a la $\mathrm{c}$ obertura de oferta educativa según el indicador propuesto, servicios educativos por cada cien habitantes de entre diez y diecinueve años en el área rural.

A partir de ello, se toma como hipótesis que el ritmo de creación de escuelas es exógeno a las características de los adolescentes en cada área rural. Considerando las características socioeconómicas relevantes, la expansión de la cobertura de la oferta educativa se aproxima a una asignación aleatoria. Esto, en términos de la literatura de evaluación de impacto de políticas públicas, equivale a que no hay selección en observables.

El indicador de cobertura de oferta educativa se utilizará como indicador de tratamiento en un marco conceptual estándar de evaluación de impacto de políticas públicas. Se le denominará indicador de intensidad del tratamiento, debido a que este indicador no divide a la muestra en grupos de tratamiento y control, sino describe el grado o nivel de acceso a los servicios educativos. Este enfoque es similar al de Duflo (2001), quien estimó los efectos de la construcción intensiva de escuelas realizada en Indonesia entre 1973 y 1978 sobre los años de educación y el salario, utilizando un indicador de tratamiento definido en función de la oferta educativa por habitante.

En la presente investigación, la evaluación se realiza mediante un método de diferencias en diferencias generalizado, teniendo como referencia metodológica —además de Duflo (2001) - , los estudios de Callaway y Sant’Anna (2018), y Besley y Burgess (2004). Basándose en especificaciones como las mencionadas por Bertrand, Duflo y Mullainathan (2004), y Hansen (2007), un método de diferencias en diferencias generalizado, con múltiples periodos y múltiples grupos puede expresarse de la siguiente manera:

$$
y_{i j t}=\alpha_{0}+\alpha_{j}+\alpha_{t}+\delta T_{j t}+X_{i j t} \beta+v_{j t}+\epsilon_{i j t}
$$

En esta fórmula, “i” es un índice por individuo, "j” es un índice por grupo $\mathrm{y}$ " $\mathrm{t}$ " es un índice de tiempo. El modelo contempla un conjunto de efectos fijos por tiempo, $\alpha_{i} ;$ efectos fijos por grupo, $\alpha_{j}$; variables de política, $T_{j t}$; covariables por individuo, $X_{i j j}$; efectos no observables por grupo/tiempo, $v_{t} ; \mathrm{y}$ errores específicos por individuo, $\epsilon_{i j t^{*}}$ 
En este caso, los grupos “j” en los que se agrupan las observaciones son los distritos y el índice de tiempo considerará el período de 2004 a 2017. Teniendo en cuenta las características de los datos y del contexto en el cual se desarrolla la intervención que analizamos, se aplican modificaciones a la expresión (1).

Debido a que la base de datos se construye sobre la base de la Encuesta de Hogares, las estimaciones incorporan el diseño muestral de Enaho para una ponderación adecuada de la importancia relativa de cada observación y el cálculo correcto de los errores estándar.

La variable de intensidad del tratamiento $\left(T_{j t}\right)$ pudo calcularse para cada año y distrito desde el Padrón de Instituciones Educativas. La implementación adecuada de la ecuación (1) requiere la inclusión de efectos fijos a nivel de distrito y de año para emular el método de diferencias en diferencias. No obstante, la estimación de efectos fijos por distritos ofrece dos tipos de dificultades: una computacional y otra estadística. En la base de datos, a razón del número de códigos ubigeo, tenemos 1874 distritos. Dicha cifra constituye una dificultad computacional, debido a que incorporar tal número de efectos fijos implicaría definir un número mayor de variables al que paquetes estadísticos como Stata pueden manejar al aplicar algoritmos de máxima verosimilitud necesarios para la estimación de modelos de probabilidad no lineal. Desde el punto de vista estadístico, la estimación de efectos fijos por distrito implicaría la estimación de parámetros para cada distrito. Sin embargo, además de la sobreparametrización del modelo, ello podría ser discutible, puesto que el mayor nivel de desagregación a nivel de estimación que permite el diseño muestral de Enaho es el departamento. Por lo expuesto, el efecto fijo espacial utilizado funciona a nivel de departamento. El supuesto subyacente es que los efectos fijos por distrito, no incluidos dentro de las variables de control, son similares dentro de cada departamento. En la sección de discusión de resultados, se desarrollan con mayor detalle los resultados que se obtendrían al considerar efectos fijos distritales.

De acuerdo con lo explorado en la literatura, la dinámica dentro del ámbito rural es heterogénea, lo cual implica la necesidad de incorporar la posibilidad de que el impacto de las políticas evaluadas sea distinto de acuerdo con el nivel de ruralidad. Esto se evalúa mediante la interacción de la variable de tratamiento con un indicador del nivel de ruralidad. Este nivel de ruralidad equivale al porcentaje de la población que habitaba en área rural en cada departamento y año. Otros estudios - Imai y Ratkovic (2013), y Vansteelandt y Goetghebeur (2003) - también han empleado este tipo de interacciones para calcular efectos de tratamiento condicionales promedio (CATE).

Dicho lo anterior, las especificaciones usadas se expresan de la siguiente forma general:

$$
y_{i j t}=f\left(\alpha_{0}+\alpha_{j}+\alpha_{t}+\delta T_{j t}+\gamma T_{j t} R_{j t}+X_{i j} \beta+\epsilon_{i j t}\right)
$$

Considerando que los índices " $\mathrm{i}$ ” y " $\mathrm{t}$ ” identifican al distrito y al año respectivamente, en (2), se establece lo siguiente: 
- $\quad y_{j t}:$ Variable dependiente ${ }^{6}$

- $\quad T_{j t}:$ Variable de intensidad de tratamiento: cantidad de servicios educativos en el área rural de nivel secundaria en el distrito por cada cien habitantes de entre diez y diecinueve años.

- $\quad R_{j i}$ : Nivel de ruralidad: porcentaje de la población que habitaba en área rural en cada departamento7 y año.

- $\quad X_{i j} \beta$ : Este miembro de la ecuación agrupa variables de control por individuo/hogar. En la mayoría de los casos, las variables usadas son la edad en años cumplidos, la falta de acceso a agua potable y electricidad, el nivel educativo de las cabezas de hogar, el sexo de la cabeza de hogar, el número de hijos en el hogar y un indicador de lengua materna indígena.

- $\delta$ : Sensibilidad de la variable dependiente a la creación de escuelas secundarias en área rural, sin considerar la interacción de la ruralidad.

- $\quad \gamma$ : Efecto interacción de la creación de escuelas secundarias y el nivel de ruralidad.

- $\quad \alpha_{0}, \alpha_{j}, \alpha_{t} y \epsilon_{i j t}$ : Constante, efecto fijo por departamento, efecto fijo temporal y término de error estocástico, respectivamente.

Las variables dependientes usadas para medir la escolaridad y la participación laboral son la matrícula en un centro educativo, la asistencia a un centro educativo ${ }^{8}$, los años de educación aprobados, la realización de algún trabajo y el total de horas trabajadas. La población bajo análisis serán los adolescentes entre catorce y diecisiete años, debido a este rango etario es potencialmente beneficiario de aumentos contemporáneos en la oferta educativa y porque el módulo de empleo de la Encuesta Nacional de Hogares recopila información para personas a partir de los catorce años.

De acuerdo con la ambigüedad encontrada en la literatura sobre una posible relación causal entre la participación laboral y la escolaridad, y que lo más razonable es pensar que ambas decisiones se toman de manera simultánea y considerando el contexto, la mayor parte de nuestras estimaciones optará por trabajar con ambas variables por separado para evitar el problema de endogeneidad. Una de las excepciones a esta consideración es la especificación de un modelo probit bivariado, en la lógica de Lokshin, Newson et al. (2011) y Greene (2012), el cual se usa para ensayar el nivel de interacción entre la escolaridad y la participación laboral adolescente.

6. Cada variable dependiente implicará una estimación por separado.

7. Debido a que esta proporción también fue estimada a partir de la Encuesta Nacional de Hogares, el mayor nivel de desagregación con el cual se puede estimar este indicador es a nivel departamental

8. La encuesta realiza esta pregunta solamente a individuos que han respondido que se encuentran matriculados en un centro educativo. Cabe anotar que la pregunta se da en términos de si actualmente asiste regularmente a un centro de enseñanza. Sin embargo, no se precisa la temporalidad específica o la definición de "regular". 
Al trabajar con variables dependientes medidas como proporción o variables dicotómicas, la estimación de modelos lineales genera riesgos típicos de este tipo de escenarios: estimaciones de $y$ fuera del intervalo [0,1], errores no normales, heteroscedasticidad, entre otros. En ese sentido, la forma funcional $f($.$) expresada en (2) asume formas no lineales de acuerdo con supuestos más$ plausibles sobre la distribución de las variables dependientes según aplique, como por ejemplo la estimación a través de funciones de probabilidad de tipo Logit o Probit.

\section{Resultados}

\section{Efecto en la matrícula y la asistencia escolar}

El primer conjunto de estimaciones se realizó para las variables de matrícula y asistencia escolar. Ambas variables son dicotómicas; por ello, la ecuación (2) se estimó mediante las especificaciones no lineales Logit y Probit, además de la forma lineal. Las variables de control corresponden a las mencionadas en la especificación de la ecuación (2) para ambos casos.

La Encuesta de Hogares registra el mes en el que se efectuó la encuesta en cada hogar. De este modo, fue posible ajustar la muestra solamente a los hogares encuestados en los meses con clases de acuerdo con el calendario escolar asumiendo lo establecido por el Ministerio de Educación, es decir, de abril a diciembre en 2004 y 2005, y de marzo a diciembre a partir del año 2006 en adelante. Se excluyen los meses de vacaciones, porque podríamos subestimar el efecto de las variables dependientes al considerar períodos en los que esperamos que la presencia de estudiantes en los centros educativos sea mínima independientemente de la oferta de instituciones educativas.

Los valores presentados en la Tabla 3 y en el resto de cuadros muestran los efectos marginales de las variables explicativas sobre las variables dependientes $(d y / d x)$. Por ejemplo, la interpretación del valor " 0,050 " de la columna (1) indica que el aumento de un centro educativo de nivel secundaria en el área rural de un distrito por cada 100 adolescentes de entre 11 y 19 años aumentaría en alrededor del 5\% la tasa de matrícula en los adolescentes de 14 a 17 años del área rural. 
Tabla 3. Efecto del aumento de la oferta educativa secundaria rural en la matrícula y asistencia escolar*

\begin{tabular}{|c|c|c|c|c|c|c|}
\hline & \multicolumn{2}{|c|}{ Modelo lineal } & \multicolumn{2}{|c|}{ Modelo Logit } & \multicolumn{2}{|c|}{ Modelo Probit } \\
\hline & $(1)$ & $(2)$ & (3) & $(4)$ & $(5)$ & (6) \\
\hline \multicolumn{7}{|c|}{ Matrícula escolar } \\
\hline \multirow[t]{2}{*}{$\mathrm{T}$} & $-0,034$ & $-0,047$ & 0,000 & $-0,070$ & $-0,002$ & $-0,054$ \\
\hline & $(0,034)$ & $(0,066)$ & $(0,043)$ & $(0,072)$ & $(0,040)$ & $(0,071)$ \\
\hline \multirow[t]{2}{*}{ TxR } & & 0,031 & & 0,172 & & 0,126 \\
\hline & & $(0,153)$ & & $(0,171)$ & & $(0,163)$ \\
\hline R2 & 0,203 & 0,203 & & & & \\
\hline Muestra & 35206 & 35206 & 34915 & 34915 & 34915 & 34915 \\
\hline \multicolumn{7}{|c|}{ Asistencia escolar } \\
\hline \multirow[t]{2}{*}{$\mathrm{T}$} & 0,004 & $-0,018$ & 0,017 & $-0,036$ & 0,017 & $-0,028$ \\
\hline & $(0,024)$ & $(0,045)$ & $(0,020)$ & $(0,028)$ & $(0,018)$ & $(0,026)$ \\
\hline \multirow[t]{2}{*}{ TxR } & & 0,055 & & $0,133^{*}$ & & $0,113^{*}$ \\
\hline & & $(0,115)$ & & $(0,076)$ & & $(0,068)$ \\
\hline R2 & 0,134 & 0,134 & & & & \\
\hline Muestra & 26479 & 26479 & 18528 & 18528 & 18528 & 18528 \\
\hline
\end{tabular}

* Nota: Todos los modelos incluyen efectos fijos por año y por departamento. Además, se incluyen como variables de control la edad en años cumplidos, la falta de acceso a agua potable y electricidad, el nivel educativo de las cabezas de hogar, el sexo de la cabeza de hogar, el número de hijos en el hogar y un indicador de lengua materna indígena. La muestra no considera respuestas realizadas en meses de vacaciones según el calendario escolar. Con el objetivo de comparabilidad, las estimaciones en Logit y Probit presentan los efectos marginales en lugar de los parámetros estimados.

Los estadísticos F representan los resultados no transformados de los modelos.

Los errores estándar se muestran en paréntesis. ${ }^{\star} \mathrm{p}<0,10 ;{ }^{* *} \mathrm{p}<0,05 ;{ }^{* *} \mathrm{p}<0,01$.

Fuente: Elaboración propia. 
Se presentan dos versiones de la estimación por cada forma funcional. La primera ofrece la estimación del efecto directo de la variable de tratamiento; y la segunda, el efecto directo más la interacción de la variable de tratamiento y el nivel de ruralidad.

En el caso de la matrícula escolar (parte superior de la tabla), las especificaciones de la primera, tercera y quinta columna muestran una relación positiva entre la creación de IIEE y la matrícula escolar. Se puede ver en la segunda, cuarta y sexta columna que, en presencia de la variable de tratamiento $T$, el término de interacción $T \times R$ no ofrece información sobre la probabilidad de matrícula. Por ese motivo, podemos interpretar que la creación de instituciones educativas de nivel secundaria en el área rural ha aumentado la probabilidad de matrícula en las zonas de área rural sin diferencias resaltantes entre el nivel de ruralidad que tiene la región. Las estimaciones en las versiones logit y probit muestran efectos marginales de menor magnitud, aunque los signos son consistentes.

En el caso de la asistencia escolar (parte inferior de la tabla), vemos que no hay efecto del tratamiento al $5 \%$ de significancia. Es posible, no obstante, que sí haya habido una mayor asistencia y que la estructura de los datos no permita que sean identificados. La Encuesta Nacional de Hogares realiza la pregunta en términos de si "actualmente asiste regularmente a un centro de enseñanza", no se precisa la temporalidad específica. Al 10\% de significancia, los efectos marginales asociados $T \times R$ dan indicios de que la asistencia pueden haber mejorado en los departamentos con mayor población rural.

\section{Efecto en años de educación alcanzados}

Un indicador directo del nivel de escolaridad es el número de años aprobados. La variable años de educación alcanzados fue calculada a partir del último año que los individuos declararon haber aprobado y se cuantificó de acuerdo con los años que implica según la duración en años que contempla la EBR en cada uno de sus niveles. En ese sentido, por ejemplo, para una persona que aprobó hasta cuarto grado de primaria, diremos que ha concluido el equivalente a siete años de educación (tres años en inicial y cuatro años en primaria); y, para una persona que completó la EBR, catorce años. Debido a que hemos acotado el análisis al rango etario de 14 a 17 años, para casi la totalidad de individuos de la muestra, la máxima cantidad de años de educación que se pudo haber alcanzado es de catorce años.

Las estimaciones del efecto del aumento de la oferta educativa en esta variable (Tabla 4) se han realizado mediante ecuaciones lineales, $y$, a diferencia de las estimaciones respecto las probabilidades de asistencia y matrícula, no se restringe la muestra a meses con clases. 
Tabla 4. Efecto del aumento de la oferta educativa secundaria rural en los años de educación total aprobados*

\begin{tabular}{|c|c|c|c|c|c|c|}
\hline & \multicolumn{2}{|c|}{ Total } & \multicolumn{2}{|c|}{ Población Masculina } & \multicolumn{2}{|c|}{ Población Femenina } \\
\hline & (1) & (2) & (3) & (4) & (5) & (6) \\
\hline \multirow[t]{2}{*}{$\mathrm{T}$} & $0,363^{* *}$ & $-0,988^{\star \star \star}$ & 0,181 & $-1,130^{\star \star \star}$ & $0,480^{* *}$ & $-0,705^{\star}$ \\
\hline & $(0,162)$ & $(0,341)$ & $(0,180)$ & $(0,355)$ & $(0,204)$ & $(0,374)$ \\
\hline \multirow[t]{2}{*}{ TxR } & & $3,300^{* * *}$ & & $3,252^{\star * *}$ & & $3,008^{\star * *}$ \\
\hline & & $(0,710)$ & & $(0,758)$ & & $(0,820)$ \\
\hline $\mathrm{F}$ & 609,856 & 559,424 & 397,601 & 365,720 & 295,499 & 270,214 \\
\hline p-valor & 0,000 & 0,000 & 0,000 & 0,000 & 0,000 & 0,000 \\
\hline R2 & 0,179 & 0,179 & 0,193 & 0,194 & 0,167 & 0,167 \\
\hline Muestra & 42833 & 42833 & 23628 & 23628 & 20829 & 20829 \\
\hline
\end{tabular}

${ }^{*}$ Nota: Las estimaciones se han obtenido a partir de un modelo de regresión lineal en todos los casos. Se incluyen efectos fijos por año y por departamento. Además de las variables de tratamiento, se incluyen como variables de control la edad en años cumplidos, la falta de acceso a agua potable y electricidad, el nivel educativo de las cabezas de hogar, el sexo de la cabeza de hogar, el número de hijos en el hogar y un indicador de lengua materna indígena.

Errores estándar en paréntesis: ${ }^{\star} \mathrm{p}<0,10 ;{ }^{* \star} \mathrm{p}<0,05 ;{ }^{* * *} \mathrm{p}<0,01$

Los efectos medidos por separado de $T$ y $T \times R$ muestran el mismo signo positivo. De acuerdo con la primera columna, un aumento de un servicio de secundaria en el área rural de un distrito por cada 100 adolescentes genera un incremento contemporáneo aproximado de 0,213 años de educación en la población rural de 14 a 17 años. Podemos ver en las columnas con rótulo (2) que, en presencia de $T \times R$, la variable $T$ pierde capacidad explicativa. A partir de ello, podemos concluir que el principal efecto del aumento de la oferta educativa en el área rural se captura mediante el término de interacción $T \times R$. El nivel de escolaridad medido en términos de años de educación aprobados ha sido más sensible al incremento de la oferta educativa en las regiones con mayores índices de ruralidad.

\section{Efecto en la probabilidad de trabajar}

La variable que captura la probabilidad de trabajar es la respuesta dicotómica a la pregunta "En la última semana, ¿ha realizado algún trabajo?". En este caso, se ha incluido efectos fijos por mes a la ecuación (2) para capturar la potencial estacionalidad que tiene el trabajo, tanto por el componente secular anual del ciclo económico como por el efecto de las vacaciones y el calendario escolar, en la medida que estamos estudiando empleo adolescente. 
En la Tabla 5, se muestran las estimaciones: para las tres formas funcionales planteadas; para la población completa, para la población masculina y para la población femenina; y considerando solo el efecto de $T$ y teniendo en cuenta el efecto simultáneo entre $T$ y $T \times R$. Las tres formas funcionales muestran efectos marginales similares, incluso en el modelo lineal en gran parte de las estimaciones. Si bien cualitativamente ofrece las mismas conclusiones, el modelo lineal sobreestima las magnitudes asociadas al efecto de la variable de tratamiento. Tal como se discutió, los modelos de probabilidad no lineal son más confiables en la medida en que sus supuestos se ajustan a la distribución de la variable dependiente.

Tabla 5. Efecto del aumento de la oferta educativa secundaria rural en la probabilidad de realizar algún trabajo*

\begin{tabular}{|c|c|c|c|c|c|c|}
\hline \multirow{2}{*}{ Modelo lin } & \multicolumn{2}{|c|}{ Total } & \multicolumn{2}{|c|}{ Población masculina } & \multicolumn{2}{|c|}{ Población femenina } \\
\hline & & & & & & \\
\hline \multirow[t]{2}{*}{$\mathrm{T}$} & -0.043 & $-0,481^{\star * *}$ & 0,001 & $-0,389^{* * *}$ & $-0,051$ & $-0,479^{* * *}$ \\
\hline & $(0.049)$ & $(0,125)$ & $(0,054)$ & $(0,130)$ & $(0,056)$ & $(0,120)$ \\
\hline \multirow[t]{2}{*}{ TxR } & & $1,092^{\star * *}$ & & $0,992^{\star * *}$ & & $1,082^{* * *}$ \\
\hline & & $(0,248)$ & & $(0,270)$ & & $(0,262)$ \\
\hline $\mathrm{F}$ & 14,403 & 15,197 & 11,998 & 12,194 & 6,338 & 7,278 \\
\hline p-valor & 0,000 & 0,000 & 0,000 & 0,000 & 0,000 & 0,000 \\
\hline $\mathrm{R} 2$ & 0,007 & 0,008 & 0,009 & 0,010 & 0,006 & 0,007 \\
\hline Muestra & 35190 & 35190 & 19541 & 19541 & 17023 & 17023 \\
\hline \multicolumn{7}{|c|}{ Modelo Logit } \\
\hline \multirow[t]{2}{*}{$\mathrm{T}$} & $-0,085$ & $-0,617^{\star * *}$ & $-0,041$ & $-0,462^{\star * *}$ & $-0,115^{*}$ & $-0,689^{* * *}$ \\
\hline & $(0,053)$ & $(0,133)$ & $(0,056)$ & $(0,152)$ & $(0,064)$ & $(0,152)$ \\
\hline \multirow[t]{2}{*}{ TxR } & & $1,255^{\star * *}$ & & $1,044^{* * *}$ & & $1,350^{* * *}$ \\
\hline & & $(0,271)$ & & $(0,307)$ & & $(0,316)$ \\
\hline Muestra & 35090 & 35090 & 19389 & 19389 & 16797 & 16797 \\
\hline \multicolumn{7}{|c|}{ Modelo Probit } \\
\hline \multirow[t]{2}{*}{$\mathrm{T}$} & $-0,080$ & $-0,521^{\star \star *}$ & $-0,042$ & $-0,387^{\star * *}$ & $-0,111^{*}$ & $-0,644^{\star * * *}$ \\
\hline & $(0,050)$ & $(0,135)$ & $(0,055)$ & $(0,125)$ & $(0,062)$ & $(0,137)$ \\
\hline \multirow[t]{2}{*}{ TxR } & & $1,109^{\star * *}$ & & $0,919^{* * *}$ & & $1,267^{\star * *}$ \\
\hline & & $(0,263)$ & & $(0,266)$ & & $(0,287)$ \\
\hline Muestra & 35090 & 35090 & 19389 & 19389 & 16797 & 16797 \\
\hline
\end{tabular}

${ }^{*}$ Nota: Se incluyen efectos fijos por año; efectos fijos por departamento; $y$, dada la ciclicidad de la variable dependiente, efectos fijos por mes. Adicionalmente, se incluyen como variables de control la edad en años cumplidos, falta de acceso a agua potable y electricidad, el nivel educativo de las cabezas de hogar, el sexo de la cabeza de hogar, el número de hijos en el hogar y un indicador de lengua materna indígena. Con el objetivo de comparabilidad, las estimaciones en Logit y Probit se presentan los efectos marginales en lugar de los parámetros estimados.

Errores estándar en paréntesis: ${ }^{*} \mathrm{p}<0,10 ;{ }^{* *} \mathrm{p}<0,05 ;{ }^{* *} \mathrm{p}<0,01$ 
En función de los resultados, se puede ver que evaluar el impacto de la oferta educativa solamente a partir del indicador $T$, sin considerar la interacción con la intensidad de la ruralidad, puede llevar concluir que la oferta educativa no ha tenido impacto en el empleo adolescente en el área rural.

La inclusión simultánea de $T$ y $T \times R$ muestra que hay dos efectos significativos, pero en direcciones opuestas en respuesta al aumento de la oferta educativa. En primera instancia, por los valores negativos estimados del efecto marginal de $T$, podemos concluir que el aumento de la oferta educativa de nivel secundaria en el área rural conduce a una reducción en la probabilidad de empleo adolescente. Sin embargo, el signo positivo del efecto marginal del término con interacción $T \times R$ indica que el efecto positivo se reduce en la medida en que existe mayor ruralidad en la región en donde se encuentra cada individuo.

Las estimaciones asociadas a la población femenina sugieren un efecto de mayor magnitud y significancia en comparación a la población total y a la población masculina. Ello puede interpretarse como una mayor sensibilidad relativa del empleo adolescente femenino ante el mayor al acceso educativo.

\section{Efecto en el total de horas dedicadas al trabajo}

El siguiente indicador analizado es el total de horas dedicadas al trabajo. Por indisponibilidad de información, estas horas de trabajo no incluyen actividades domésticas. Los resultados se presentan en la Tabla 6. La estimación presentada en la parte superior se ha realizado con las variables de control y los efectos fijos de las estimaciones anteriores. Con esta especificación, solo es posible concluir efectos de la variable tratamiento en la población masculina. A partir de ello, se puede concluir que no hay evidencia de cambios en la duración de la jornada de trabajo de las adolescentes de 14 a 17 años del área rural que participan en el mercado de trabajo como respuesta al aumento de los servicios educativos. Es decir, la respuesta se concentra en la participación en el trabajo. 
Tabla 6. Efecto del aumento de la oferta educativa secundaria rural en el total de horas trabajadas*

\begin{tabular}{|c|c|c|c|c|c|c|}
\hline & \multicolumn{2}{|c|}{ Total } & \multicolumn{2}{|c|}{ Población masculina } & \multicolumn{2}{|c|}{ Población femenina } \\
\hline & (1) & (2) & (3) & (4) & (5) & (6) \\
\hline \multirow[t]{2}{*}{$\mathrm{T}$} & $-1,573$ & $-5,940^{\star *}$ & $-2,104$ & $-8,359^{\star * *}$ & $-1,836$ & $-2,496$ \\
\hline & $(1,397)$ & $(2,787)$ & $(1,560)$ & $(3,230)$ & $(1,915)$ & $(3,371)$ \\
\hline \multirow[t]{2}{*}{ TxR } & & $10,454^{*}$ & & $16,016^{\star *}$ & & 1,527 \\
\hline & & $(5,936)$ & & $(6,598)$ & & $(7,536)$ \\
\hline F & 85,013 & 77,753 & 58,109 & 53,210 & 33,950 & 31,071 \\
\hline P-valor & 0,000 & 0,000 & 0,000 & 0,000 & 0,000 & 0,000 \\
\hline R2 & 0,097 & 0,097 & 0,126 & 0,126 & 0,088 & 0,088 \\
\hline Muestra & 27839 & 27839 & 16160 & 16160 & 12757 & 12757 \\
\hline
\end{tabular}

${ }^{*}$ Nota: Además de las variables de tratamiento, se incluyen como variables de control la edad en años cumplidos, la falta de acceso a agua potable y electricidad, el nivel educativo de las cabezas de hogar, el sexo de la cabeza de hogar, el número de hijos en el hogar y un indicador de lengua materna indígena. Se incluyen efectos fijos por año; efectos fijos por departamento; $y$, dada la ciclicidad de la variable dependiente, efectos fijos por mes.

Errores estándar en paréntesis: ${ }^{\star} \mathrm{p}<0,10 ;{ }^{* *} \mathrm{p}<0,05 ;{ }^{* * *} \mathrm{p}<0,01$

\section{Efecto conjunto en la participación escolar y laboral}

Un enfoque que permite la evaluación conjunta de la participación escolar y la prevalencia del empleo adolescente es el uso de modelos Probit bivariados. Para ello, redefinimos el indicador de participación laboral como la "no realización de un trabajo". En este caso, la ecuación (2) se convierte en un sistema de ecuaciones del siguiente tipo:

$$
\begin{aligned}
& P 1_{i j t}=\phi\left(a_{0}^{1}+\alpha_{j}^{1}+\alpha_{t}^{1}+\delta^{1} T_{j t}+\gamma^{1} T_{j t} R_{j t}+X_{j} \beta^{1}+\epsilon_{i j t}^{1}\right) \\
& P 2_{i j t}=\phi\left(a_{0}^{2}+\alpha_{j}^{2}+\alpha_{t}^{2}+\delta^{2} T_{j t}+\gamma^{2} T_{j t} R_{j t}+X_{j} \beta^{2}+\epsilon_{i j t}^{2}\right)
\end{aligned}
$$


Ambas relaciones se determinan de manera conjunta en su componente estocástico:

$$
\left(\begin{array}{c}
\epsilon^{1} \\
\epsilon^{2}
\end{array} \mid T, T \times R, X\right) \sim N\left[\left(\begin{array}{l}
0 \\
0
\end{array}\right),\left(\begin{array}{ll}
1 & \rho \\
\rho & 1
\end{array}\right)\right]
$$

Este enfoque ofrece un marco de referencia para estimar relaciones causales de factores exógenos que influyen en dos variables que se determinan de manera conjunta, y, adicionalmente, permite calcular la correlación condicional entre ambas variables. En este caso, elegimos las variables P1, probabilidad de no realizar ningún trabajo, y $\mathrm{P} 2$, la probabilidad de matrícula en un centro educativo o la probabilidad de asistir regularmente a un centro educativo en el cual se encuentre matriculado (ambas definiciones por separado). Las tablas 7 y 8 contienen los resultados de este ejercicio.

Las estimaciones correspondientes a la matrícula a un centro educativo coinciden con los enfoques de secciones anteriores. Adicionalmente, se identifica que el efecto es de menor magnitud para la población masculina. Las estimaciones sobre la probabilidad de no realizar ningún trabajo también coinciden con las estimaciones realizadas previamente, con excepción de la submuestra masculina, en que el parámetro asociado al efecto es menor. Además, la estimación del coeficiente de correlación condicional $\rho^{h}$ evidencia una decisión simultánea entre la matrícula en un centro educativo y la no realización de trabajo adolescente, la cual tiene mayor magnitud en la población masculina. La tercera sección de la Tabla 7 muestra el efecto marginal medio del aumento de la oferta educativa en la probabilidad conjunta de no trabajar y asistir a un centro educativo. Los resultados indican que el aumento de los servicios educativos ha reconfigurado la situación de la población adolescente femenina rural en mayor medida que a la masculina, tanto en términos de acceso a los servicios educativos como en cuanto a la reducción de la prevalencia del trabajo. 
Tabla 7. Efecto del aumento de la oferta educativa en la probabilidad conjunta de no realizar un empleo y estar matriculado en un centro educativo*

\begin{tabular}{|c|c|c|c|c|c|c|}
\hline & \multicolumn{2}{|c|}{ Total } & \multicolumn{2}{|c|}{ Población masculina } & \multicolumn{2}{|c|}{ Población femenina } \\
\hline & (1) & (2) & (3) & (4) & (5) & (6) \\
\hline \multicolumn{7}{|c|}{ P1: No realizó ningún trabajo } \\
\hline \multirow[t]{2}{*}{$\mathrm{T}$} & 0,224 & $1,511^{\star \star *}$ & 0,120 & $1,168^{\star \star *}$ & $0,320^{*}$ & $1,884^{* * *}$ \\
\hline & $(0,143)$ & $(0,389)$ & $(0,166)$ & $(0,378)$ & $(0,181)$ & $(0,399)$ \\
\hline \multirow[t]{2}{*}{$\mathrm{T} \times \mathrm{R}$} & & $-3,237^{\star * *}$ & & $-2,795^{\star * *}$ & & $-3,722^{* * *}$ \\
\hline & & $(0,761)$ & & $(0,806)$ & & $(0,834)$ \\
\hline \multicolumn{7}{|c|}{ P2: Está matriculado en un centro de enseñanza regular } \\
\hline \multirow[t]{2}{*}{$\mathrm{T}$} & 0,038 & $-0,322$ & 0,075 & $-0,093$ & $-0,012$ & $-0,503^{*}$ \\
\hline & $(0,157)$ & $(0,246)$ & $(0,221)$ & $(0,355)$ & $(0,192)$ & $(0,294)$ \\
\hline \multirow[t]{2}{*}{$\mathrm{T} \times \mathrm{R}$} & & 0,873 & & 0,390 & & 1,264 \\
\hline & & $(0,602)$ & & $(0,795)$ & & $(0,773)$ \\
\hline \multirow[t]{2}{*}{$\rho^{h}$} & $0,172^{\star \star *}$ & $0,173^{* * *}$ & $0,242^{* * *}$ & $0,243^{\star * *}$ & $0,163^{\star * *}$ & $0,164^{\star * *}$ \\
\hline & $(0,014)$ & $(0,014)$ & $(0,019)$ & $(0,019)$ & $(0,019)$ & $(0,019)$ \\
\hline \multicolumn{7}{|c|}{ Efecto marginal medio: $\operatorname{Pr}(\mathrm{P} 1=1, \mathrm{P} 2=1)$} \\
\hline \multirow[t]{2}{*}{$\mathrm{T}$} & 0,062 & $0,368^{\star * *}$ & 0,037 & $0,301^{\star * *}$ & 0,077 & $0,404^{\star * *}$ \\
\hline & $(0,040)$ & $(0,104)$ & $(0,046)$ & $(0,103)$ & $(0,049)$ & $(0,101)$ \\
\hline \multirow[t]{2}{*}{$\mathrm{T} \times \mathrm{R}$} & & $-0,772^{\star * *}$ & & $-0,709^{* * *}$ & & $-0,769^{* * *}$ \\
\hline & & $(0,206)$ & & $(0,216)$ & & $(0,219)$ \\
\hline Muestra & 35190 & 35190 & 19541 & 19541 & 17023 & 17023 \\
\hline
\end{tabular}

${ }^{\star}$ Nota: Las estimaciones correspondientes a los modelos (1) y (2) se realizaron mediante un modelo Probit bivariado. Además de las variables de tratamiento, se incluyen como variables de control la edad en años cumplidos, la falta de acceso a agua potable y electricidad, el nivel educativo de las cabezas de hogar, el sexo de la cabeza de hogar, el número de hijos en el hogar y un indicador de lengua materna indígena. Se incluyen efectos fijos por año; efectos fijos por departamento; y, dada la ciclicidad del empleo, efectos fijos por mes.

Errores estándar en paréntesis: ${ }^{\star} \mathrm{p}<0,10 ;{ }^{* *} \mathrm{p}<0,05 ;{ }^{* * *} \mathrm{p}<0,01$

Los resultados de la Tabla 8 corresponden a la consideración de la variable asistencia a un centro educativo en lugar de la matrícula. Este procedimiento acota la muestra a los adolescentes que registran matrícula en un centro educativo y permite evaluar el efecto en la participación efectiva en clases. Los resultados correspondientes a la participación en el mercado laboral coinciden con los encontrados en la Tabla 7, con excepción de la submuestra masculina, 
en que no se encuentran efectos. No obstante, no se encuentra efectos del aumento de los servicios educativos sobre la probabilidad de asistencia. Asimismo, la estimación del coeficiente de correlación condicional $\rho^{h}$ sugiere la existencia de una decisión simultánea entre la asistencia a un centro educativo y la no realización de trabajo adolescente. No obstante, esta correlación condicional es menor para la población femenina. Al igual que en la Tabla 7, la tercera sección de la Tabla 8 sugiere que el aumento de los servicios educativos ha reconfigurado la situación de la población adolescente femenina rural en mayor medida que la de la masculina, en términos de asistencia a los centros educativos y a la participación en el mercado laboral.

Tabla 8. Efecto del aumento de la oferta educativa en la probabilidad conjunta de no realizar un empleo y asistir a un centro educativo*

\begin{tabular}{|c|c|c|c|c|c|c|}
\hline & \multicolumn{2}{|l|}{ Total } & \multicolumn{2}{|c|}{ Población masculina } & \multicolumn{2}{|c|}{ Población femenina } \\
\hline & $(1)$ & $(2)$ & (3) & $(4)$ & $(5)$ & $(6)$ \\
\hline \multicolumn{7}{|c|}{ P1: No realizó ningún trabajo } \\
\hline $\mathrm{T}$ & $\begin{array}{c}0,221 \\
(0,153)\end{array}$ & $\begin{array}{c}1,308^{* * *} \\
(0,411)\end{array}$ & $\begin{array}{c}0,199 \\
(0,180)\end{array}$ & $\begin{array}{c}1,182^{\star * *} \\
(0,441)\end{array}$ & $\begin{array}{c}0,287 \\
(0,190)\end{array}$ & $\begin{array}{c}1,477^{\star * *} \\
(0,411)\end{array}$ \\
\hline $\mathrm{T} \times \mathrm{R}$ & & $\begin{array}{c}-2,769^{\star * *} \\
(0,806)\end{array}$ & & $\begin{array}{c}-2,652^{\star * *} \\
(0,916)\end{array}$ & & $\begin{array}{c}-2,855^{\star * *} \\
(0,866) \\
\end{array}$ \\
\hline \multicolumn{7}{|c|}{ P2: Asiste regularmente a un centro educativo } \\
\hline $\mathrm{T}$ & $\begin{array}{c}0,322 \\
(0,271)\end{array}$ & $\begin{array}{c}-0,046 \\
(0,358)\end{array}$ & $\begin{array}{c}0,303 \\
(0,402)\end{array}$ & $\begin{array}{c}0,472 \\
(0,802)\end{array}$ & $\begin{array}{c}0,297 \\
(0,298)\end{array}$ & $\begin{array}{l}-0,232 \\
(0,364)\end{array}$ \\
\hline $\mathrm{T} \times \mathrm{R}$ & & $\begin{array}{c}0,868 \\
(0,927)\end{array}$ & & $\begin{array}{c}-0,424 \\
(1,538)\end{array}$ & & $\begin{array}{c}1,530 \\
(1,180)\end{array}$ \\
\hline$\rho^{h}$ & $\begin{array}{c}0,146^{* * *} \\
(0.026)\end{array}$ & $\begin{array}{c}0,145^{\text {}} \\
(0.026)\end{array}$ & $\begin{array}{c}0,247^{\star * *} \\
(0.037)\end{array}$ & $\begin{array}{c}0,247^{\star * *} \\
(0.037)\end{array}$ & $\begin{array}{l}0,069^{*} \\
(0.038) \\
\end{array}$ & $\begin{array}{l}0,069^{*} \\
(0.038)\end{array}$ \\
\hline \multicolumn{7}{|c|}{ Efecto marginal medio: $\operatorname{Pr}(\mathrm{P} 1=1, \mathrm{P} 2=1)$} \\
\hline $\mathrm{T}$ & $\begin{array}{c}0.081 \\
(0.051)\end{array}$ & $\begin{array}{c}0.429^{\star * *} \\
(0.135)\end{array}$ & $\begin{array}{c}0.069 \\
(0.058)\end{array}$ & $\begin{array}{c}0.384^{\star * *} \\
(0.142)\end{array}$ & $\begin{array}{c}0.098 \\
(0.061)\end{array}$ & $\begin{array}{c}0.456^{* * *} \\
(0.128)\end{array}$ \\
\hline $\mathrm{T} \times \mathrm{R}$ & & $\begin{array}{c}-0.889^{\star * *} \\
(0.263)\end{array}$ & & $\begin{array}{c}-0.850^{\star * *} \\
(0.293)\end{array}$ & & $\begin{array}{c}-0.852^{* * *} \\
(0.270)\end{array}$ \\
\hline Muestra & 26467 & 26467 & 15093 & 15093 & 12439 & 12439 \\
\hline
\end{tabular}

${ }^{\star}$ Nota: Solamente las personas que señalan estar matriculadas en un centro educativo responden a la pregunta correspondiente a asistencia. Las estimaciones correspondientes a los modelos (1) y (2) se realizaron mediante un modelo Probit bivariado. Además de las variables de tratamiento, se incluyen como variables de control la edad en años cumplidos, la falta de acceso a agua potable y electricidad, el nivel educativo de las cabezas de hogar, el sexo de la cabeza de hogar, el número de hijos en el hogar y un indicador de lengua materna indígena. Se incluyen efectos fijos por año; efectos fijos por departamento; y, dada la ciclicidad del empleo, efectos fijos por mes.

Errores estándar en paréntesis: ${ }^{\star} \mathrm{p}<0,10 ;{ }^{* *} \mathrm{p}<0,05 ;{ }^{* *} \mathrm{p}<0,01$ 


\section{Discusión de resultados}

Los hallazgos previos tienen limitaciones potenciales que deben ser discutidas. En primer lugar, la estimación de efectos fijos se hizo a nivel departamental para considerar el nivel de inferencia permitido por la Enaho y evitar la sobreparametrización del modelo. La agregación departamental de los efectos fijos implica el riesgo de agrupar poblaciones heterogéneas. En segundo lugar, la aplicación rigurosa del método de diferencias en diferencias requiere que los efectos fijos estén al mismo nivel en el que varía el tratamiento, en este caso, a nivel distrital. La Tabla 9 resume los resultados que se habrían encontrado utilizando efectos fijos a nivel distrital en un modelo de regresión lineal.

Además de las consideraciones discutidas en la sección de metodología, este ejercicio permite ver que la inclusión de efectos fijos a nivel de distrito genera un problema adicional al reducir considerablemente la muestra. Debido a que la Enaho está diseñada para tener representatividad a nivel de departamento, en muchos casos, la cantidad de observaciones en cada distrito es muy pequeña. Como resultado, en distritos con muy reducida cantidad de observaciones, las variables dependientes tienen muy poca o nula varianza dentro de cada grupo y son predichas perfectamente por los efectos fijos. Como consecuencia, las observaciones en esos grupos son excluidas. La muestra resultante no necesariamente tiene la misma representatividad nacional que la utilizada en el resto del documento.

Sin embargo, los resultados de la Tabla 9 constituyen una prueba adicional de los hallazgos principales del documento. Los efectos de la expansión de la oferta educativa son mayores para la población femenina en términos del incremento de su nivel educativo y la reducción de la posibilidad de trabajar. Los resultados educativos son más favorables en las áreas de mayor ruralidad, pero más desfavorables en lo que respecta a los resultados en participación laboral. Los efectos en la población masculina, además de ser de menor magnitud que en el caso femenino, no persisten al incluir efectos fijos por distrito. No obstante, se mantiene el resultado de que el efecto en años de educación es mayor en regiones con mayor población rural. Estos hallazgos corroboran la presencia de diferencias de género previamente documentadas en la literatura (Li y Liu, 2014; Jacoby y Mansuri, 2011), y sugieren que el efecto del acceso a servicios educativos es mayor en poblaciones para las cuales el costo de estudiar lejos del hogar es mayor. 
Tabla 9. Resultados con efectos fijos a nivel distrital*

\begin{tabular}{|c|c|c|c|c|c|c|c|}
\hline & \multicolumn{2}{|c|}{ Efecto promedio } & \multicolumn{4}{|c|}{ Efectos heterogéneos } & \multirow[b]{2}{*}{ Muestra } \\
\hline & $\mathrm{T}$ & & $\mathrm{T}$ & & $\mathrm{T} \times \mathrm{R}$ & & \\
\hline \multicolumn{8}{|l|}{ Población masculina } \\
\hline Matrícula escolar & 0,047 & $(0,043)$ & 0,129 & $(0,113)$ & $-0,135$ & $(0,188)$ & 15948 \\
\hline Asistencia escolar & 0,007 & $(0,026)$ & 0,012 & $(0,088)$ & $-0,008$ & $(0,133)$ & 12488 \\
\hline $\begin{array}{l}\text { Años de educación } \\
\text { total aprobados }\end{array}$ & 0,199 & $(0,172)$ & $-0,875^{\star *}$ & $(0,410)$ & $1,728^{\star * *}$ & $(0,654)$ & 23628 \\
\hline $\begin{array}{l}\text { Probabilidad de } \\
\text { trabajar }\end{array}$ & $-0,057$ & $(0,066)$ & $-0,175$ & $(0,144)$ & 0,202 & $(0,208)$ & 15945 \\
\hline Horas de trabajo & $-1,237$ & $(1,162)$ & 2,727 & $(3,713)$ & $-6,772$ & $(6,161)$ & 13236 \\
\hline \multicolumn{8}{|l|}{ Población femenina } \\
\hline Matrícula escolar & $-0,018$ & $(0,045)$ & 0,030 & $(0,121)$ & $-0,076$ & $(0,186)$ & 13916 \\
\hline Asistencia escolar & 0,025 & $(0,030)$ & 0,083 & $(0,094)$ & $-0,094$ & $(0,138)$ & 10492 \\
\hline $\begin{array}{l}\text { Años de educación } \\
\text { total aprobados }\end{array}$ & $0,351^{\star *}$ & $(0,173)$ & $-1,605^{\star * *}$ & $(0,396)$ & $3,139^{* * *}$ & $(0,661)$ & 20828 \\
\hline $\begin{array}{l}\text { Probabilidad de } \\
\text { trabajar }\end{array}$ & $-0,170^{* * *}$ & $(0,060)$ & $-0,487^{\star * *}$ & $(0,183)$ & $0,502^{*}$ & $(0,261)$ & 13912 \\
\hline Horas de trabajo & $-1,505$ & $(2,064)$ & 3,056 & $(5,717)$ & $-6,920$ & $(8,141)$ & 10576 \\
\hline
\end{tabular}

${ }^{*}$ Nota: Las estimaciones se han obtenido a partir de un modelo de regresión lineal en todos los casos. Se incluyen efectos fijos por año; por distrito; y, en el caso de las variables asociadas a la participación laboral, efectos fijos por mes. Además de las variables de tratamiento, se incluyen como variables de control la edad en años cumplidos, la falta de acceso a agua potable y electricidad, el nivel educativo de las cabezas de hogar, el sexo de la cabeza de hogar, el número de hijos en el hogar y un indicador de lengua materna indígena.

Errores estándar en paréntesis: ${ }^{\star} \mathrm{p}<0,10{ }^{* \star} \mathrm{p}<0,05 ;{ }^{* \star *} \mathrm{p}<0,01$

Por otro lado, el supuesto explícito del documento es que, por lo menos, no hay selección en observables. Una posible prueba de robustez adicional podría ser la inclusión de variables instrumentales para reducir el sesgo posible por selección en no observables. Si bien este procedimiento permitiría garantizar con mayor seguridad la dirección causal de las relaciones encontradas, la información disponible no facilita encontrar instrumentos adecuados. 
Un riesgo adicional para el análisis es la migración endógena. Si los hogares anticipan efectos de los servicios educativos lo suficientemente favorables, ello podría influir en sus decisiones de migración. La inclusión de esta dimensión en el análisis es complicada, debido a que la migración interdistrital está disponible solo a partir de 2014. No obstante, la información disponible muestra que la migración de los adolescentes en el rango etario considerado, sobre todo en el área rural, es baja.

Tabla 10. Migración en adolescentes de 14 a 17 años

\begin{tabular}{lcccc}
\hline & \multicolumn{4}{c}{ Años } \\
\cline { 2 - 5 } & 2014 & 2015 & 2016 & 2017 \\
\hline Hace cinco años vivía en este distrito & & & \\
\hline Proporción área rural & $96,24 \%$ & $96,96 \%$ & $96,88 \%$ & $97,52 \%$ \\
Proporción área urbana & $92,00 \%$ & $93,24 \%$ & $92,27 \%$ & $93,82 \%$ \\
\hline & $2004-2006$ & $2007-2009$ & $2011-2014$ & $2015-2017$ \\
\hline Es parte del hogar & & & & \\
\hline Proporción área rural & $97,36 \%$ & $96,98 \%$ & $97,27 \%$ & $97,66 \%$ \\
Proporción área urbana & $97,09 \%$ & $96,69 \%$ & $97,36 \%$ & $97,77 \%$ \\
\hline
\end{tabular}

Fuente: Enaho 2004-2017. Elaboración propia.

Futuras investigaciones que utilicen otras fuentes de información o apliquen métodos experimentales o cualitativos podrían contribuir a verificar los resultados de los problemas discutidos e incluir dimensiones no consideradas en el presente documento. Entre la agenda pendiente, se requiere analizar con mayor detalle el hecho de que las adolescentes tienen mayor carga de actividades domésticas no remuneradas, y que ello puede influir en sus resultados laborales y educativos. Según los resultados de la ETI 2015 (OIT y MTPE, 2016), el porcentaje de niñas y adolescentes que estudian y realizan actividades domésticas (50,0\%) es mayor al de sus pares varones (40,1\%).

Si bien en este documento se ha encontrado evidencia del vínculo contemporáneo entre la oferta educativa y el trabajo adolescente, la literatura no aborda con suficiente claridad los mecanismos detrás del trabajo infantil y adolescente, como indican Basu, Das y Dutta (2010), incluidas sus posibles respuestas a la oferta educativa. Dentro de la agenda teórica que complementa los resultados empíricos de este documento, se encuentra el modelamiento específico del mercado de trabajo infantil, lo que incluye su relación con la participación educativa. 


\section{Conclusiones}

En este estudio, se documenta la expansión de la oferta educativa secundaria en el área rural, y se exploran sus efectos en la escolaridad y el empleo adolescente en los hogares mediante un enfoque de diferencias en diferencias generalizado, utilizando los datos de la Encuesta Nacional de Hogares entre los años 2004 y 2017, y el Padrón de Instituciones Educativas de 2017. Los resultados sugieren heterogeneidad en cuanto al nivel de ruralidad de las regiones y el género de los adolescentes.

Se ha encontrado que el aumento de los servicios educativos tiene efectos positivos en la tasa de matrícula, en los años de educación alcanzados y en la reducción de la posibilidad de participar en el mercado de trabajo. No se ha hallado, sin embargo, evidencia de una reducción en la jornada laboral de los adolescentes que participan del mercado de trabajo. En general, los efectos para la población femenina son mayores y persisten ante la inclusión de efectos fijos a nivel de distrito. Además, los resultados educativos han sido más favorables para los adolescentes en regiones con mayor población rural, a pesar de que en estas regiones la prevalencia de trabajo adolescente, en ambos sexos, y su respuesta a la oferta educativa es menor.

Pese a los posibles sesgos de género en las familias, los resultados de este documento muestran que las condiciones externas a los hogares explican un componente significativo del rezago educativo femenino respecto al masculino particularmente en años anteriores. La provisión de servicios educativos que estén al alcance de poblaciones vulnerables, particularmente en el área rural, es importante para promover la participación universal de los adolescentes en el sistema educativo. En ese sentido, los hallazgos de este documento muestran la pertinencia de acciones como la Política de Atención Educativa para la Población del Ámbito Rural. ${ }^{9}$

Si bien la atención educativa es importante para mejorar los indicadores de escolaridad, requiere una estrategia multisectorial. Este documento muestra evidencia de que la participación educativa y el trabajo adolescente se determina de manera simultánea. En paralelo a los esfuerzos del sector educación, son necesarias estrategias focalizadas a la reducción de la demanda por trabajo infantil y adolescente. Por ejemplo, fortalecer el apoyo a la actividad agropecuaria debido a que es la actividad predominante del área rural, de los hogares en situación de pobreza y en la que se desenvuelve la mayor parte de los adolescentes trabajadores.

9. Decreto Supremo que aprueba la Política de Atención Educativa para la Población de Ámbitos Rurales. Decreto Supremo No 013-2018-MINEDU, 14 de diciembre de 2018 


\section{Agradecimientos}

Se agradecen, especialmente, los comentarios y sugerencias de los participantes de los talleres correspondientes al programa de becas, en especial al Dr. César Guadalupe. El trabajo se benefició también de los comentarios de colegas del Centro de Investigación de la Universidad del Pacífico, en particular del Dr. Jorge Dávalos, y de los aportes valiosos de dos revisores anónimos. 


\section{Referencias bibliográficas}

Alcázar, L. (2009). Asistencia y deserción en escuelas secundarias rurales del Perú. Revista Iberoamericana sobre Calidad, Eficaciay Cambio en Educación, 7(4). Recuperado de https://repositorio.uam.es/handle/10486/661242

Alcázar, L., Rendón, S., y Wachtenheim, E. (2002). Working and studying in rural latin america: Critical decisions of adolescence. IDB Working Paper No. 177. Recuperado de https://ssrn.com/abstract $=1814727$

Basu, K., Das, S. y Dutta, B. (2010). Child labor and household wealth: Theory and empirical evidence of an inverted-U. Journal of Development Economics, 91(1), 8-14.

Beegle, K., Dehejia, R., y Gatti, R. (2006). Child labor and agricultural shocks. Journal of Development Economics, 81(1). Recuperado de https://www. sciencedirect.com/science/article/pii/S0304387805000829

(2009). Why should we care about child labor? The education, labor market, and health consequences of child labor. The Journal of Human Resources, 44(4). Recuperado de http://jhr. uwpress.org/content/44/4/871.short

Bertrand, M., Duflo, E., y Mullainathan, S. (2004). How much should we trust differences-indifferences estimates? The Quarterly journal of economics, 119(1), 249-275. Recuperado de https://academic.oup.com/qje/ article/119/1/249/1876068

Besley, T., y Burgess, R. (2004). Can labor regulation hinder economic performance? evidence from India. The Quarterly Journal of Economics, 119(1). Recuperado de https://academic.oup.com/qje/ article/119/1/91/1876083

Boyd, C. (2014). Decisiones de inserción laboral: El caso de los jóvenes rurales peruanos. Economía, 37(74). Recuperado de http://revistas.pucp.edu.pe/ index.php/economia/article/view/11412

Callaway, B., y Sant'Anna, P. (2018). Difference-in-differences with multiple time periods and an application on the minimum wage and employment. No publicado. Recuperado de https://arxiv.org/abs/1803.09015

Chacaltana, J. (2012). Transiciones juveniles y trayectoria laboral: El caso peruano. Lima: OIT, UNFPA, OIM, PNUD.

Consejo Nacional de Educación (2006). Proyecto educativo nacional al 2021: La educación que queremos para el Perú. Lima: Consejo Nacional de Educación.

Cueto, S. (2004). Factores predictivos del rendimiento escolar, deserción e ingreso a educación secundaria en una muestra de estudiantes de zonas rurales del Perú. Education Policy Analysis Archives, 12(35). Recuperado de http://epaa.asu.edu/epaa/v12n35/ 
Dasgupta, P. (2007). Nutritional status, the capacity for work and poverty traps. Journal of Econometrics, 77(1). Recuperado de https://www.sciencedirect. com/science/article/pii/S0304407696018040

Dizon-Ross, R. (2019). Parents' beliefs about their children's academic ability: Implications for educational investments. American Economic Review, 109(8), 2728-65. Recuperado de https://www.aeaweb.org/ articles?id=10.1257/aer.20171172

Duflo, E. (2001). Schooling and labor market consequences of school construction in Indonesia: Evidence from an unusual policy experiment. American Economic Review, 91(4). Recuperado de https://www.aeaweb. org/articles?id=10.1257/aer.91.4.795

Emerson, P. M., y Souza, A. P. (2007). Child labor, school attendance, and intrahousehold gender bias in Brazil. The World Bank Economic Review, 21(2). Recuperado de https://academic.oup.com/wber/ article/21/2/301/1916067

Greene, W. H. (2012). Econometric analysis. $7^{\text {th }}$ ed. New York: Prentice Hall.

Hansen, C. B. (2007). Generalized least squares inference in panel and multilevel models with serial correlation and fixed effects. Journal of Econometrics, 140(2), 670-694. Recuperado de https://www.sciencedirect.com/science/ article/pii/S0304407606001515

Imai, K., y Ratkovic, M. (2013). Estimating treatment effect heterogeneity in randomized program evaluation. Annals of Applied Statistics, 7(1). Recuperado de: https://projecteuclid.org/euclid.aoas/1365527206

International Programme on the Elimination of Child Labour (2004). Investing in every child: An economic study of the costs and benefits of eliminating child labour. ILO/IPEC. Recuperado de http://white.lim.ilo.org/ipec/ documentos/costos_y_beneficios_eliminacion_ti_oit_2004.pdf

Instituto Nacional de Estadística e Informática (2007). Censos Nacionales 2007: XI de Población y VI de Vivienda. Lima: Instituto Nacional de Estadística e Informática (INEI).

(2016a). Alrededor de 2 millones de niñas, niños y adolescentes trabajan en el país (Nota de prensa). Inei.gob. Recuperado de https://www.inei.gob.pe/prensa/noticias/alrededor-de-2millones-de-ninas-ninos-y-adolescentes-trabajan-en-el-pais-9394/

(2016b). Población total al 30 de junio, por grupos quinquenales de edad, según departamento, provincia y distrito, 2005-2015. Inei.gob. https://www.inei.gob.pe/media/ MenuRecursivo/indices_tematicos/cuadro001_1.xls

XII de Población y VII de Vivienda. Lima: Instituto Nacional de Estadística e Informática. 
Izzo, C. V., Weissberg, R. P., Kasprow, W. J., y Fendrich, M. (1999). A longitudinal assessment of teacher perceptions of parent involvement in children's education and school performance. American Journal of Community Psychology, 27(6), 817-839. Recuperado de https:/onlinelibrary.wiley. com/doi/abs/10.1023/A:1022262625984

Jacoby, H. G., y Mansuri, G. (2011). Crossing boundaries: Gender, caste and schooling in rural Pakistan. Policy Research Working Paper 5710. The World Bank.

Knight, J., Shi, L., y Quheng, D. (2010). Education and the poverty trap in rural China: Closing the trap. Oxford Development Studies, 30(1). Recuperado de https://www.tandfonline.com/doi/abs/10.1080/13600810903551595?c asa_token=0qvqob6AyREAAAAA:eizVBik0VoBexVCrLji0Nsy7fXRawF 5o_bCO6P7SVwNL5qkjDt-KrZXrqOJYacmoX7xd2tSP4hgvhUs

Li, L. y Liu, H. (2014). Primary school availability and middle school education in rural China. Labour Economics, 28, 24-40. Recuperado de https://www. sciencedirect.com/science/article/pii/S092753711400027X

Lokshin, M., Newson, R. B (2011). Impact of interventions on discrete outcomes: Maximum likelihood estimation of the binary choice models with binary endogenous regressors. Stata Journal, 11(3), 368-385. Recuperado de https://journals.sagepub.com/doi/pdf/10.1177/1536867X1101100303

Marchetta, F., Sahn, D., y Tiberti, L. (2018). The role of weather shocks in Madagascar. Études er Documents Cerdi, 2018(2), 1-60. Recuperado de: https://papers.ssrn.com/sol3/papers.cfm?abstract_id=3266899

Nielsen, H. (1998). Child labor and school attendance: Two joint decisions. (Working Paper No. 98-15). Recuperado de https://papers.ssrn.com/sol3/ papers.cfm?abstract_id $=176068$

Ravallion, M., y Wodon, Q. (2000). Does child labour displace schooling? Evidence on behavioural responses to an enrollment subsidy. The Economic Journal, 110(March). Recuperado de https://onlinelibrary.wiley. com/doi/abs/10.1111/1468-0297.00527

Organización Internacional del Trabajo, y Ministerio del Trabajo y Promoción del Empleo (2016). Magnitud y características del trabajo infantil en Perú. Informe de 2015. Lima: OIT y MTPE. Recuperado de: http://white.lim.ilo. org/ipec/documentos/informeti_2015_peru.pdf

Sistema de Información de Tendencias Educativas en América Latina. (2010). Trabajo adolescente y escolarización. Dato Destacado (17). Recuperado: http://archivo.siteal.iipe.unesc o.org/sites/default/files/ datodestacado_20101115.pdf

Song, L., Appleton, S. y Knight, J. (2006). Why do girls in rural China have lower school enrollment? World Development, 34(9), 1639-1653. Recuperado de https://www.sciencedirect.com/science/article/pii/S0305750X06001094 
Urueña, S., Tovar, L. M., y Castillo, M. (2009). Determinantes del trabajo infantil y la escolaridad: el caso del valle del Cauca en Colombia. Revista Latinoamericana de Ciencias Sociales, Niñez y Juventud, 7(2), 707-733. Recuperado de http://vitela.javerianacali.edu.co/handle/11522/3541

Van der Berg, S. Burger, C., Burger, R., de Vos, M., du Rand, G., Gustafsson, M., Moses, E., Shepherd, D. L., Spaull, N., Taylor, S., van Broekhuizen, H., y Fintel, D. (2011). Low quality education as a poverty trap (Stellenbosch Economic Working Papers No. 25). Recuperado de: https://papers.ssrn. com/sol3/papers.cfm?abstract_id=2973766

Vansteelandt, S., y Goetghebeur,E. (2003). Causal inference with generalized structural mean models. Journal of the Royal Statistical Society: Series B, 65(4). Recuperado de https://rss.onlinelibrary.wiley.com/doi/abs/10.1046/ j.1369-7412.2003.00417.x

Winter, N. (2001). CORR_SVY: Stata module to compute correlation tables for survey data. 